\title{
Ramanujan-Fourier series of certain arithmetic functions of two variables
}

\author{
Noboru Ushiroya
}

\begin{abstract}
We study Ramanujan-Fourier series of certain arithmetic functions of two variables. We generalize Delange's theorem to the case of arithmetic functions of two variables and give sufficient conditions for pointwise convergence of Ramanujan-Fourier series of arithmetic functions of two variables. We also give several examples which are not obtained by trivial generalizations of results on Ramanujan-Fourier series of functions of one variable.

Keywords. Ramanujan-Fourier series, two variables, arithmetic functions, multiplicative functions.
\end{abstract}

2010 Mathematics Subject Classification. 11A25, 11N37

\section{Introduction}

Let $c_{q}(n)$ denote the Ramanujan sums defined in [Ra18] as

$$
c_{q}(n)=\sum_{\substack{a=1 \\(a, q)=1}}^{q} \exp (2 \pi i a n / q),
$$

where $q$ and $n$ are positive integers and $(a, q)$ is the greatest common divisor of $a$ and $q$. Ramanujan proved that $c_{q}(n)$ can be rewritten as

$$
c_{q}(n)=\sum_{d \mid(q, n)} \mu(q / d) d
$$

where $\mu$ is the Möbius function. Hardy [Ha21] proved that, for fixed $n, c_{q}(n)$ is a multiplicative function. In other words,

$$
c_{q_{1} q_{2}}(n)=c_{q_{1}}(n) c_{q_{2}}(n)
$$

holds for any $q_{1}, q_{2} \in \mathbb{N}$ satisfying $\left(q_{1}, q_{2}\right)=1$. Let $f: \mathbb{N} \mapsto \mathbb{C}$ be an arithmetic function. Ramanujan [Ra18] investigated its Ramanujan-Fourier series which is an infinite series of the form

$$
f(n) \sim \sum_{q=1}^{\infty} a_{q} c_{q}(n)
$$

where $a_{q}$ are called the Ramanujan-Fourier coefficients of $f$, and he obtained the following results.

$$
\begin{aligned}
& \frac{\sigma_{s}(n)}{n^{s}}=\zeta(s+1) \sum_{q=1}^{\infty} \frac{c_{q}(n)}{q^{s+1}}, \\
& \frac{\varphi(n)}{n}=\frac{1}{\zeta(2)} \sum_{q=1}^{\infty} \frac{\mu(q)}{\varphi_{2}(q)} c_{q}(n), \\
& \tau(n)=-\sum_{q=1}^{\infty} \frac{\log q}{q} c_{q}(n), \\
& r(n)=\pi \sum_{q=1}^{\infty} \frac{(-1)^{q-1}}{2 q-1} c_{2 q-1}(n),
\end{aligned}
$$

We thank episciences.org for providing open access hosting of the electronic journal Hardy-Ramanujan Journal 
where $\sigma_{s}(n)=\sum_{d \mid n} d^{s}$ with $s>0, \quad \zeta(s)$ is the Riemann zeta function, $\varphi(n)$ is Euler's totient function, $\varphi_{s}(n)=n^{s} \prod_{p \mid n}\left(1-1 / p^{s}\right), \tau(n)$ is the number of divisors of $n$ and $r(n)$ is the number of representations of $n$ as the sum of two squares.

Let $*$ denotes the Dirichlet convolution, that is, $(f * g)(n)=\sum_{d \mid n} f(d) g(n / d)$ for arithmetic functions $f, g$, and let $\omega(n)$ be the number of distinct prime divisors of $n$. We say that $f: \mathbb{N} \mapsto \mathbb{C}$ is a multiplicative function if $f$ satisfies $f(m n)=f(m) f(n)$ for any $m, n \in \mathbb{N}$ satisfying $(m, n)=1$.

Delange [De76] proved the following theorem.

Theorem 1.1. ([De76]) Let $f(n)$ be an arithmetic function satisfying

$$
\sum_{n=1}^{\infty} 2^{\omega(n)} \frac{|(f * \mu)(n)|}{n}<\infty
$$

Then its Ramanujan-Fourier series is pointwise convergent and

$$
f(n)=\sum_{q=1}^{\infty} a_{q} c_{q}(n)
$$

holds where

$$
a_{q}=\sum_{m=1}^{\infty} \frac{(f * \mu)(q m)}{q m} .
$$

Moreover, if $f$ is a multiplicative function, then $a_{q}$ can be rewritten as

$$
a_{q}=\prod_{p \in \mathcal{P}}\left(\sum_{e=\nu_{p}(q)}^{\infty} \frac{(f * \mu)\left(p^{e}\right)}{p^{e}}\right)
$$

where $\mathcal{P}$ is the set of prime numbers and $\nu_{p}(q)=\left\{\begin{array}{lll}\alpha & \text { if } & p^{\alpha} \| n \\ 0 & \text { if } & p \nmid n .\end{array}\right.$

Delange noted that, if $f$ is a multiplicative function, then the condition (1.6) is equivalent to the condition: $\sum_{p \in \mathcal{P}} \sum_{e=1}^{\infty}\left|f\left(p^{e}\right)-f\left(p^{e-1}\right)\right| / p^{e}<\infty$ since $(f * \mu)\left(p^{e}\right)=f\left(p^{e}\right)-f\left(p^{e-1}\right)$ for $e \geqq 1$. Under this condition, we can directly calculate Ramanujan-Fourier coefficients $a_{q}$ for certain arithmetic functions by using (1.7). For example, if we set $f(n)=\varphi(n) / n$, then we can easily calculate the righthand side of (1.7) and obtain $a_{q}=(\zeta(2))^{-1} \mu(q) / \varphi_{2}(q)$ which coincide with the Ramanujan-Fourier coefficients of (1.3).

Many results concerning Ramanujan-Fourier series of arithmetic functions of one variable are obtained by many mathematicians hitherto, however, as for Ramanujan-Fourier series of arithmetic functions of two variables, to my knowledge, few results are known. We would like to extend Delange's theorem to the case of functions of two variables and obtain several examples which are extensions of $(1.2) \sim(1.5)$.

\section{Some Results}

Let $f, g: \mathbb{N} \times \mathbb{N} \mapsto \mathbb{C}$ be arithmetic functions of two variables. The Dirichlet convolution of $f$ and $g$ is defined as follows.

$$
(f * g)\left(n_{1}, n_{2}\right)=\sum_{m_{1}\left|n_{1}, m_{2}\right| n_{2}} f\left(m_{1}, m_{2}\right) g\left(n_{1} / m_{1}, n_{2} / m_{2}\right) .
$$

We use the same notation $\mu$ for the function

$$
\mu\left(n_{1}, n_{2}\right)=\mu\left(n_{1}\right) \mu\left(n_{2}\right),
$$


which is the inverse of the constant function 1 under the Dirichlet convolution, that is, $(\mu * 1)\left(n_{1}, n_{2}\right)=$ $\delta\left(n_{1}, n_{2}\right)$ holds where $\delta\left(n_{1}, n_{2}\right)=1$ or 0 according to whether $n_{1}=n_{2}=1$ or not.

We investigate Ramanujan-Fourier series of arithmetical functions of two variables along Delange's article ([De76]). We first establish the following theorem which is an extension of Theorem 1.1 to the case of arithmetic functions of two variables.

Theorem 2.1. Let $f\left(n_{1}, n_{2}\right)$ be an arithmetic function of two variables satisfying

$$
\sum_{n_{1}, n_{2}=1}^{\infty} 2^{\omega\left(n_{1}\right)} 2^{\omega\left(n_{2}\right)} \frac{\left|(f * \mu)\left(n_{1}, n_{2}\right)\right|}{n_{1} n_{2}}<\infty .
$$

Then its Ramanujan-Fourier series is pointwise convergent and

$$
f\left(n_{1}, n_{2}\right)=\sum_{q_{1}, q_{2}=1}^{\infty} a_{q_{1}, q_{2}} c_{q_{1}}\left(n_{1}\right) c_{q_{2}}\left(n_{2}\right)
$$

holds where

$$
a_{q_{1}, q_{2}}=\sum_{m_{1}, m_{2}=1}^{\infty} \frac{(f * \mu)\left(m_{1} q_{1}, m_{2} q_{2}\right)}{m_{1} q_{1} m_{2} q_{2}}
$$

For the proof of the above theorem, we need the following lemma.

Lemma 2.2. ([De76]) For every positive integer $k$,

$$
\sum_{q \mid k}\left|c_{q}(n)\right| \leqq n 2^{\omega(k)}
$$

Proof of Theorem 2.1. We proceed as in [De76]. We first note that (2.8) implies the absolute convergence of the right-hand side of (2.10). Next we show that the series $\sum_{q_{1}, q_{2}=1}^{\infty} a_{q_{1}, q_{2}} c_{q_{1}}\left(n_{1}\right) c_{q_{2}}\left(n_{2}\right)$ is absolutely convergent. It is easy to see that

$$
\begin{aligned}
\sum_{q_{1}, q_{2}=1}^{\infty}\left|a_{q_{1}, q_{2}} c_{q_{1}}\left(n_{1}\right) c_{q_{2}}\left(n_{2}\right)\right| & \leqq \sum_{q_{1}, q_{2}=1}^{\infty} \sum_{m_{1}, m_{2}=1}^{\infty}\left|\frac{(f * \mu)\left(q_{1} m_{1}, q_{2} m_{2}\right)}{q_{1} m_{1} q_{2} m_{2}} c_{q_{1}}\left(n_{1}\right) c_{q_{2}}\left(n_{2}\right)\right| \\
& =\sum_{k_{1}, k_{2}=1}^{\infty} W_{k_{1}, k_{2}},
\end{aligned}
$$

where

$$
W_{k_{1}, k_{2}}=\sum_{\substack{m_{1} q_{1}=k_{1} \\ m_{2} q_{2}=k_{2}}}\left|\frac{(f * \mu)\left(q_{1} m_{1}, q_{2} m_{2}\right)}{q_{1} m_{1} q_{2} m_{2}} c_{q_{1}}\left(n_{1}\right) c_{q_{2}}\left(n_{2}\right)\right|=\left|\frac{(f * \mu)\left(k_{1}, k_{2}\right)}{k_{1} k_{2}}\right| \sum_{\substack{q_{1}\left|k_{1} \\ q_{2}\right| k_{2}}}\left|c_{q_{1}}\left(n_{1}\right) c_{q_{2}}\left(n_{2}\right)\right| .
$$

By (2.8) and Lemma 2.2 we have

$$
\sum_{k_{1}, k_{2}=1}^{\infty} W_{k_{1}, k_{2}} \leqq \sum_{k_{1}, k_{2}=1}^{\infty}\left|\frac{(f * \mu)\left(k_{1}, k_{2}\right)}{k_{1} k_{2}}\right| n_{1} n_{2} 2^{\omega\left(k_{1}\right)} 2^{\omega\left(k_{2}\right)} \ll n_{1} n_{2}<\infty .
$$

Hence the series $\sum_{q_{1}, q_{2}=1}^{\infty} a_{q_{1}, q_{2}} c_{q_{1}}\left(n_{1}\right) c_{q_{2}}\left(n_{2}\right)$ is absolutely convergent. We have

$$
\sum_{q_{1}, q_{2}=1}^{\infty} \sum_{m_{1}, m_{2}=1}^{\infty} \frac{(f * \mu)\left(q_{1} m_{1}, q_{2} m_{2}\right)}{q_{1} m_{1} q_{2} m_{2}} c_{q_{1}}\left(n_{1}\right) c_{q_{2}}\left(n_{2}\right)=\sum_{k_{1}, k_{2}=1}^{\infty} w_{k_{1}, k_{2}},
$$


where

$$
w_{k_{1}, k_{2}}=\sum_{\substack{m_{1} q_{1}=k_{1} \\ m_{2} q_{2}=k_{2}}} \frac{(f * \mu)\left(q_{1} m_{1}, q_{2} m_{2}\right)}{q_{1} m_{1} q_{2} m_{2}} c_{q_{1}}\left(n_{1}\right) c_{q_{2}}\left(n_{2}\right)=\frac{(f * \mu)\left(k_{1}, k_{2}\right)}{k_{1} k_{2}} \sum_{\substack{q_{1}\left|k_{1} \\ q_{2}\right| k_{2}}} c_{q_{1}}\left(n_{1}\right) c_{q_{2}}\left(n_{2}\right) .
$$

Using the well known formula: $\sum_{q \mid k} c_{q}(n)=\varepsilon_{k}(n)$ where $\varepsilon_{k}(n)=\left\{\begin{array}{lll}k & \text { if } & k \mid n \\ 0 & \text { if } & k \nmid n\end{array}\right.$ ([Si89]), we have

$$
\begin{aligned}
\sum_{k_{1}, k_{2}=1}^{\infty} w_{k_{1}, k_{2}} & =\sum_{k_{1}, k_{2}=1}^{\infty} \frac{(f * \mu)\left(k_{1}, k_{2}\right)}{k_{1} k_{2}} \varepsilon_{k_{1}}\left(n_{1}\right) \varepsilon_{k_{2}}\left(n_{2}\right) \\
& =\sum_{\substack{k_{1}\left|n_{1} \\
k_{2}\right| n_{2}}} \frac{(f * \mu)\left(k_{1}, k_{2}\right)}{k_{1} k_{2}} k_{1} k_{2}=(f * \mu * 1)\left(n_{1}, n_{2}\right)=(f * \delta)\left(n_{1}, n_{2}\right)=f\left(n_{1}, n_{2}\right) .
\end{aligned}
$$

Therefore (2.9) holds. This completes the proof of Theorem 2.1.

We say that $f: \mathbb{N} \times \mathbb{N} \mapsto \mathbb{C}$ is a multiplicative function of two variables if $f$ satisfies

$$
f\left(m_{1} n_{1}, m_{2} n_{2}\right)=f\left(m_{1}, m_{2}\right) f\left(n_{1}, n_{2}\right)
$$

for any $m_{1}, m_{2}, n_{1}, n_{2} \in \mathbb{N}$ satisfying $\left(m_{1} m_{2}, n_{1} n_{2}\right)=1$. It is well known that if $f$ and $g$ are multiplicative functions of two variables, then $f * g$ also becomes a multiplicative function of two variables. For an arithmetical function $f$ of two variable, the mean value $M(f)$ is defined by

$$
M(f)=\lim _{x, y \rightarrow \infty} \frac{1}{x y} \sum_{n_{1} \leqq x, n_{2} \leqq y} f\left(n_{1}, n_{2}\right)
$$

if this limit exists. Ushiroya [Us07] proved the following theorem.

Theorem 2.3. ([Us07]) Let $f$ be a multiplicative function of two variables satisfying

$$
\sum_{p \in \mathcal{P}} \sum_{\substack{e_{1}, e_{2} \geqq 0 \\ e_{1}+e_{2} \geqq 1}} \frac{\left|(f * \mu)\left(p^{e_{1}}, p^{e_{2}}\right)\right|}{p^{e_{1}+e_{2}}}<\infty .
$$

Then the mean value $M(f)$ exists and

$$
M(f)=\sum_{m_{1}, m_{2}=1}^{\infty} \frac{(f * \mu)\left(m_{1}, m_{2}\right)}{m_{1} m_{2}}=\prod_{p \in \mathcal{P}}\left(\sum_{e_{1}, e_{2}=0}^{\infty} \frac{(f * \mu)\left(p^{e_{1}}, p^{e_{2}}\right)}{p^{e_{1}+e_{2}}}\right) .
$$

We would like to investigate Ramanujan-Fourier series in the case when $f$ is a multiplicative function of two variables. The following theorem is an extension of Theorem 1.1 to the case of a multiplicative function of two variables.

Theorem 2.4. Let $f$ be a multiplicative function of two variables satisfying

$$
\sum_{p \in \mathcal{P}} \sum_{\substack{e_{1}, e_{2} \geqq 0 \\ e_{1}+e_{2} \geqq 1}} \frac{\left|(f * \mu)\left(p^{e_{1}}, p^{e_{2}}\right)\right|}{p^{e_{1}+e_{2}}}<\infty .
$$

Then its Ramanujan-Fourier series is pointwise convergent and

$$
f\left(n_{1}, n_{2}\right)=\sum_{q_{1}, q_{2}=1}^{\infty} a_{q_{1}, q_{2}} c_{q_{1}}\left(n_{1}\right) c_{q_{2}}\left(n_{2}\right)
$$


holds where

$$
a_{q_{1}, q_{2}}=\sum_{m_{1}, m_{2}=1}^{\infty} \frac{(f * \mu)\left(m_{1} q_{1}, m_{2} q_{2}\right)}{m_{1} q_{1} m_{2} q_{2}}
$$

Moreover, if $M(f) \neq 0$ and $\left\{q_{1}, q_{2}\right\}>1$, where $\left\{q_{1}, q_{2}\right\}$ denotes the least common multiple of $q_{1}$ and $q_{2}$, then $a_{q_{1}, q_{2}}$ can be rewritten as follows.

$$
\begin{aligned}
a_{q_{1}, q_{2}} & =\prod_{p \in \mathcal{P}}\left(\sum_{e_{1}=\nu_{p}\left(q_{1}\right)} \sum_{e_{2}=\nu_{p}\left(q_{2}\right)} \frac{(f * \mu)\left(p^{e_{1}}, p^{e_{2}}\right)}{p^{e_{1}+e_{2}}}\right) \\
& =M(f) \prod_{p \mid\left\{q_{1}, q_{2}\right\}}\left\{\left(\sum_{e_{1}=\nu_{p}\left(q_{1}\right)} \sum_{e_{2}=\nu_{p}\left(q_{2}\right)} \frac{(f * \mu)\left(p^{e_{1}}, p^{e_{2}}\right)}{p^{e_{1}+e_{2}}}\right) /\left(\sum_{e_{1}=0} \sum_{e_{2}=0} \frac{(f * \mu)\left(p^{e_{1}}, p^{e_{2}}\right)}{p^{e_{1}+e_{2}}}\right)\right\},
\end{aligned}
$$

Remark 2.5. By (2.12) and (2.15), we have $a_{1,1}=M(f)$.

Proof. First we note that, if $f\left(n_{1}, n_{2}\right)$ is a multiplicative function of two variables, then $f\left(n_{1}, n_{2}\right)=$ $\prod_{p \in \mathcal{P}} f\left(p^{\nu_{p}\left(n_{1}\right)}, p^{\nu_{p}\left(n_{2}\right)}\right)$ holds. Let $f$ satisfy $(2.13)$. Since $\left(n_{1}, n_{2}\right) \mapsto 2^{\omega\left(n_{1}\right)} 2^{\omega\left(n_{2}\right)}\left|(f * \mu)\left(n_{1}, n_{2}\right)\right| / n_{1} n_{2}$ is a multiplicative function of two variables, we have

$$
\begin{aligned}
& \sum_{n_{1} \leqq x, n_{2} \leqq y} 2^{\omega\left(n_{1}\right)} 2^{\omega\left(n_{2}\right)} \frac{\left|(f * \mu)\left(n_{1}, n_{2}\right)\right|}{n_{1} n_{2}} \leqq \sum_{k, \ell \geqq 0} \prod_{p \in \mathcal{P}} 2^{\omega\left(p^{k}\right)} 2^{\omega\left(p^{\ell}\right)} \frac{\left|(f * \mu)\left(p^{k}, p^{\ell}\right)\right|}{p^{k+\ell}} \\
\ll & \prod_{p \in \mathcal{P}} \sum_{k, \ell \geqq 0} \frac{\left|(f * \mu)\left(p^{k}, p^{\ell}\right)\right|}{p^{k+\ell}}=\prod_{p \in \mathcal{P}}\left(1+\sum_{\substack{e_{1}, e_{2} \geqq 0 \\
e_{1}+e_{2} \geqq 1}} \frac{\left|(f * \mu)\left(p^{e_{1}}, p^{e_{2}}\right)\right|}{p^{e_{1}+e_{2}}}\right) \\
\leqq & \exp \left(\sum_{p \in \mathcal{P}} \sum_{\substack{e_{1}, e_{2} \geqq 0 \\
e_{1}+e_{2} \geqq 1}} \frac{\left|(f * \mu)\left(p^{e_{1}}, p^{e_{2}}\right)\right|}{p^{e_{1}+e_{2}}}\right)<\infty
\end{aligned}
$$

where we have used the well-known inequality $1+x \leqq \exp (x)$ for $x \geqq 0$. Since $f$ satisfies $(2.8)$, we see by Theorem 2.1 that the Ramanujan-Fourier series of $f$ is pointwise convergent and (2.14) holds.

Next we prove (2.16) under the condition $M(f) \neq 0$ and $\left\{q_{1}, q_{2}\right\}>1$. For $i=1,2$, let $q_{i}=\prod_{j} p_{j}^{e_{i j}}$ $\left(e_{i j} \geqq 0\right)$ and $m_{i}=r_{i} \prod_{j} p_{j}^{d_{i j}}\left(d_{i j} \geqq 0\right)$ be the prime factor decompositions of $q_{i}$ and $m_{i}$ respectively where $p_{j}$ 's are prime numbers satisfying $p_{j} \mid\left\{q_{1}, q_{2}\right\}$ and $r_{i}$ 's are positive integers coprime to $q_{1} q_{2}$. Then we have

$$
a_{q_{1}, q_{2}}=\sum_{m_{1}, m_{2}=1}^{\infty} \frac{(f * \mu)\left(m_{1} q_{1}, m_{2} q_{2}\right)}{m_{1} q_{1} m_{2} q_{2}}=\sum_{\substack{d_{i j} \geqq 0 \\ r_{i} \geqq 1,\left(r_{i}, q_{1} q_{2}\right)=1}} \frac{(f * \mu)\left(r_{1} \prod_{j} p_{j}^{d_{1 j}+e_{1 j}}, r_{2} \prod_{j} p_{j}^{d_{2 j}+e_{2 j}}\right)}{r_{1} r_{2} \prod_{j} p_{j}^{d_{1 j}+e_{1 j}+d_{2 j}+e_{2 j}}} .
$$

Since $f * \mu$ is multiplicative, we have

$$
\begin{aligned}
& a_{q_{1}, q_{2}}=\sum_{\substack{d_{i j} \geqq 0 \\
r_{i} \geqq 1,\left(r_{i}, q_{1} q_{2}\right)=1}} \frac{(f * \mu)\left(\prod_{j} p_{j}^{d_{1 j}+e_{1 j}}, \prod_{j} p^{d_{2 j}+e_{2 j}}\right)}{\prod_{j} p_{j}^{d_{1 j}+e_{1 j}+d_{2 j}+e_{2 j}}} \times \frac{(f * \mu)\left(r_{1}, r_{2}\right)}{r_{1} r_{2}} \\
& =\left(\sum_{d_{i j} \geqq 0} \frac{(f * \mu)\left(\prod_{j} p_{j}^{d_{1 j}+e_{1 j}}, \prod_{j} p^{d_{2 j}+e_{2 j}}\right)}{\prod_{j} p_{j}^{d_{1 j}+e_{1 j}+d_{2 j}+e_{2 j}}}\right)\left(\sum_{r_{i} \geqq 1,\left(r_{i}, q_{1} q_{2}\right)=1} \frac{(f * \mu)\left(r_{1}, r_{2}\right)}{r_{1} r_{2}}\right) \\
& =\prod_{p \mid\left\{q_{1}, q_{2}\right\}}\left(\sum_{e_{1}=\nu_{p}\left(q_{1}\right)} \sum_{e_{2}=\nu_{p}\left(q_{2}\right)} \frac{(f * \mu)\left(p^{e_{1}}, p^{e_{2}}\right)}{p^{e_{1}+e_{2}}}\right) \times \prod_{p \nmid q_{1} q_{2}}\left(\sum_{e_{1}=0} \sum_{e_{2}=0} \frac{(f * \mu)\left(p^{e_{1}}, p^{e_{2}}\right)}{p^{e_{1}+e_{2}}}\right) .
\end{aligned}
$$


Since the condition $M(f) \neq 0$ implies $\sum_{e_{1}, e_{2}=0} \frac{(f * \mu)\left(p^{e_{1}}, p^{e_{2}}\right)}{p^{e_{1}+e_{2}}} \neq 0$ for every $p \in \mathcal{P}$ by $(2.12)$, we have

$$
\begin{aligned}
a_{q_{1}, q_{2}}= & \frac{\prod_{p \mid\left\{q_{1}, q_{2}\right\}}\left(\sum_{e_{1}=\nu_{p}\left(q_{1}\right)} \sum_{e_{2}=\nu_{p}\left(q_{2}\right)} \frac{(f * \mu)\left(p^{e_{1}}, p^{e_{2}}\right)}{p^{e_{1}+e_{2}}}\right) \prod_{p \in \mathcal{P}}\left(\sum_{e_{1}=0} \sum_{e_{2}=0} \frac{(f * \mu)\left(p^{e_{1}}, p^{e_{2}}\right)}{p^{e_{1}+e_{2}}}\right)}{\prod_{p \mid\left\{q_{1}, q_{2}\right\}}\left(\sum_{e_{1}=0} \sum_{e_{2}=0} \frac{(f * \mu)\left(p^{e_{1}}, p^{e_{2}}\right)}{p^{e_{1}+e_{2}}}\right)} \\
= & M(f) \prod_{p \mid\left\{q_{1}, q_{2}\right\}}\left\{\left(\sum_{e_{1}=\nu_{p}\left(q_{1}\right)} \sum_{e_{2}=\nu_{p}\left(q_{2}\right)} \frac{(f * \mu)\left(p^{e_{1}}, p^{e_{2}}\right)}{p^{e_{1}+e_{2}}}\right) /\left(\sum_{e_{1}=0} \sum_{e_{2}=0} \frac{(f * \mu)\left(p^{e_{1}}, p^{e_{2}}\right)}{p^{e_{1}+e_{2}}}\right)\right\} .
\end{aligned}
$$

This completes the proof of Theorem 2.4.

Next we consider the case when $f$ is represented in the form $f\left(n_{1}, n_{2}\right)=g\left(n_{1} n_{2}\right)$ where $g$ is a multiplicative function of one variable. We begin with the following lemma.

Lemma 2.6. Let $f\left(n_{1}, n_{2}\right)=g\left(n_{1} n_{2}\right)$ where $g$ is a multiplicative function satisfying

$$
\sum_{p \in P}\left(\sum_{e \geqq 1} \frac{\left|g\left(p^{e}\right)-g\left(p^{e-1}\right)\right|}{p^{e}}+\sum_{e_{1}, e_{2}=1}^{\infty} \frac{\left|g\left(p^{e_{1}+e_{2}}\right)-2 g\left(p^{e_{1}+e_{2}-1}\right)+g\left(p^{e_{1}+e_{2}-2}\right)\right|}{p^{e_{1}+e_{2}}}\right)<\infty .
$$

Then the mean value $M(f)$ exists and

$$
M(f)=\prod_{p \in P}\left(1+2 \sum_{e \geqq 1} \frac{g\left(p^{e}\right)-g\left(p^{e-1}\right)}{p^{e}}+\sum_{e_{1}, e_{2}=1}^{\infty} \frac{g\left(p^{e_{1}+e_{2}}\right)-2 g\left(p^{e_{1}+e_{2}-1}\right)+g\left(p^{e_{1}+e_{2}-2}\right)}{p^{e_{1}+e_{2}}}\right) .
$$

Proof. It is easy to see that

$$
\begin{aligned}
(f * \mu)\left(p^{e}, 1\right) & =f\left(p^{e}, 1\right)-f\left(p^{e-1}, 1\right)=g\left(p^{e}\right)-g\left(p^{e-1}\right) \quad \text { if } \quad e \geqq 1, \\
(f * \mu)\left(p^{e_{1}}, p^{e_{2}}\right) & =f\left(p^{e_{1}}, p^{e_{2}}\right)-f\left(p^{e_{1}-1}, p^{e_{2}}\right)-f\left(p^{e_{1}}, p^{e_{2}-1}\right)+f\left(p^{e_{1}-1}, p^{e_{2}-1}\right) \\
& =g\left(p^{e_{1}+e_{2}}\right)-2 g\left(p^{e_{1}+e_{2}-1}\right)+g\left(p^{e_{1}+e_{2}-2}\right) \quad \text { if } \quad e_{1}, e_{2} \geqq 1 .
\end{aligned}
$$

From (2.17), (2.19) and (2.20), it is also easy to see that (2.11) holds. Therefore Lemma 2.6 follows by Theorem 2.3. This complete the proof of Lemma 2.6 .

Now we can prove the following theorem.

Theorem 2.7. Let $f\left(n_{1}, n_{2}\right)=g\left(n_{1} n_{2}\right)$ where $g$ is a multiplicative function satisfying (2.17). Then its Ramanujan-Fourier series is pointwise convergent and

$$
f\left(n_{1}, n_{2}\right)=\sum_{q_{1}, q_{2}=1}^{\infty} a_{q_{1}, q_{2}} c_{q_{1}}\left(n_{1}\right) c_{q_{2}}\left(n_{2}\right)
$$

holds where

$$
a_{q_{1}, q_{2}}=\sum_{m_{1}, m_{2}=1}^{\infty} \frac{(f * \mu)\left(m_{1} q_{1}, m_{2} q_{2}\right)}{m_{1} q_{1} m_{2} q_{2}} .
$$


Moreover, if $M(f) \neq 0, q, q_{1}, q_{2}>1$ and $\left\{q_{1}, q_{2}\right\}>1$, then $a_{q, 1}, a_{1, q}$ and $a_{q_{1}, q_{2}}$ can be rewritten as follows.

$$
\begin{aligned}
& a_{q, 1}=a_{1, q}=M(f) \prod_{p \mid q} \frac{\sum_{e=\nu_{p}(q)}^{\infty} \frac{g\left(p^{e}\right)-g\left(p^{e-1}\right)}{p^{e}}+\sum_{e_{1}=\nu_{p}(q)}^{\infty} \sum_{e_{2}=1}^{\infty} \frac{g\left(p^{e_{1}+e_{2}}\right)-2 g\left(p^{e_{1}+e_{2}-1}\right)+g\left(p^{e_{1}+e_{2}-2}\right)}{p^{e_{1}+e_{2}}}}{1+2 \sum_{e=1}^{\infty} \frac{g\left(p^{e}\right)-g\left(p^{e-1}\right)}{p^{e}}+\sum_{e_{1}, e_{2}=1}^{\infty} \frac{g\left(p^{e_{1}+e_{2}}\right)-2 g\left(p^{e_{1}+e_{2}-1}\right)+g\left(p^{e_{1}+e_{2}-2}\right)}{p^{e_{1}+e_{2}}}}, \\
& a_{q_{1}, q_{2}}=M(f) \prod_{p \mid\left\{q_{1}, q_{2}\right\}} \frac{\sum_{e_{1}=\nu_{p}\left(q_{1}\right)}^{\infty} \sum_{e_{2}=\nu_{p}\left(q_{2}\right)}^{\infty} \frac{g\left(p^{e_{1}+e_{2}}\right)-2 g\left(p^{e_{1}+e_{2}-1}\right)+g\left(p^{e_{1}+e_{2}-2}\right)}{p^{e_{1}+e_{2}}}}{p^{e}} \frac{g\left(p^{e}\right)-g\left(p^{e-1}\right)}{p^{\infty}}+\sum_{e_{1}, e_{2}=1}^{\infty} \frac{g\left(p^{e_{1}+e_{2}}\right)-2 g\left(p^{e_{1}+e_{2}-1}\right)+g\left(p^{e_{1}+e_{2}-2}\right)}{p^{e_{1}+e_{2}}} .
\end{aligned}
$$

Proof. Since (2.17), (2.19) and (2.20) implies (2.13), Theorem 2.7 holds by Theorem 2.4. We note that if $q_{2}=1$, then $\sum_{e_{1}=\nu_{p}\left(q_{1}\right)} \sum_{e_{2}=\nu_{p}\left(q_{2}\right)} \frac{(f * \mu)\left(p^{e_{1}}, p^{e_{2}}\right)}{p^{e_{1}+e_{2}}}$ in $(2.16)$ can be rewritten as

$$
\begin{aligned}
& \sum_{e_{1}=\nu_{p}\left(q_{1}\right)} \sum_{e_{2}=0} \frac{(f * \mu)\left(p^{e_{1}}, p^{e_{2}}\right)}{p^{e_{1}+e_{2}}}=\sum_{e_{1}=\nu_{p}\left(q_{1}\right)} \frac{(f * \mu)\left(p^{e_{1}}, 1\right)}{p^{e_{1}}}+\sum_{e_{1}=\nu_{p}\left(q_{1}\right)} \sum_{e_{2} \geqq 1} \frac{(f * \mu)\left(p^{e_{1}}, p^{e_{2}}\right)}{p^{e_{1}+e_{2}}} \\
= & \sum_{e=\nu_{p}\left(q_{1}\right)}^{\infty} \frac{g\left(p^{e}\right)-g\left(p^{e-1}\right)}{p^{e}}+\sum_{e_{1}=\nu_{p}\left(q_{1}\right)}^{\infty} \sum_{e_{2}=1}^{\infty} \frac{g\left(p^{e_{1}+e_{2}}\right)-2 g\left(p^{e_{1}+e_{2}-1}\right)+g\left(p^{e_{1}+e_{2}-2}\right)}{p^{e_{1}+e_{2}}} .
\end{aligned}
$$

This complete the proof of Theorem 2.7.

Next we consider the case when $f$ is represented in the form $f\left(n_{1}, n_{2}\right)=g\left(\left(n_{1}, n_{2}\right)\right)$ where $g$ is a multiplicative function of one variable. Before describing Ramanujan-Fourier series of this case, we would like to cite the following theorem.

Theorem 2.8. ([Us07]) Let $f\left(n_{1}, n_{2}\right)=g\left(\left(n_{1}, n_{2}\right)\right)$ where $g$ is a multiplicative function satisfying

$$
\sum_{p \in P} \sum_{e \geqq 1} \frac{\left|g\left(p^{e}\right)-g\left(p^{e-1}\right)\right|}{p^{2 e}}<\infty .
$$

Then the mean value $M(f)$ exists and

$$
M(f)=\prod_{p \in \mathcal{P}}\left(1+\sum_{e=1}^{\infty} \frac{g\left(p^{e}\right)-g\left(p^{e-1}\right)}{p^{2 e}}\right) .
$$

Remark 2.9. We note that, if $f\left(n_{1}, n_{2}\right)=g\left(\left(n_{1}, n_{2}\right)\right)$, then it is easy to see that

$$
(f * \mu)\left(p^{k}, p^{\ell}\right)= \begin{cases}g\left(p^{k}\right)-g\left(p^{k-1}\right) & \text { if } k=\ell \geqq 1 \\ 0 & \text { otherwise. }\end{cases}
$$

Hence (2.21) clearly implies (2.13).

Now we can show the following theorem concerning Ramanujan-Fourier series in the case $f\left(n_{1}, n_{2}\right)=$ $g\left(\left(n_{1}, n_{2}\right)\right)$. Let $k \vee \ell$ denote $\max (k, \ell)$. 
Theorem 2.10. Let $f\left(n_{1}, n_{2}\right)=g\left(\left(n_{1}, n_{2}\right)\right)$ where $g$ is a multiplicative function satisfying

$$
\sum_{p \in P} \sum_{e \geqq 1} \frac{\left|g\left(p^{e}\right)-g\left(p^{e-1}\right)\right|}{p^{2 e}}<\infty
$$

Then its Ramanujan-Fourier series is pointwise convergent and

$$
f\left(n_{1}, n_{2}\right)=\sum_{q_{1}, q_{2}=1}^{\infty} a_{q_{1}, q_{2}} c_{q_{1}}\left(n_{1}\right) c_{q_{2}}\left(n_{2}\right)
$$

holds where

$$
a_{q_{1}, q_{2}}=\sum_{m_{1}, m_{2}=1}^{\infty} \frac{(f * \mu)\left(m_{1} q_{1}, m_{2} q_{2}\right)}{m_{1} q_{1} m_{2} q_{2}} .
$$

Moreover, if $M(f) \neq 0$ and $\left\{q_{1}, q_{2}\right\}>1$, then $a_{q_{1}, q_{2}}$ can be rewritten as follows.

$$
a_{q_{1}, q_{2}}=M(f) \prod_{p \mid\left\{q_{1}, q_{2}\right\}}\left\{\left(\sum_{e=\nu_{p}\left(q_{1}\right) \vee \nu_{p}\left(q_{2}\right)}^{\infty} \frac{g\left(p^{e}\right)-g\left(p^{e-1}\right)}{p^{2 e}}\right) /\left(1+\sum_{e=1}^{\infty} \frac{g\left(p^{e}\right)-g\left(p^{e-1}\right)}{p^{2 e}}\right)\right\} .
$$

Proof. Remark 2.9 says that (2.24) implies (2.13). Therefore the Ramanujan-Fourier series of $f$ is pointwise convergent by Theorem 2.4. It is easy to see that (2.25) holds by (2.16) and (2.23). This completes the proof of Theorem 2.10 .

Next we consider the case when $f$ is represented in the form $f\left(n_{1}, n_{2}\right)=g\left(\left\{n_{1}, n_{2}\right\}\right)$ where $g$ is a multiplicative function of one variable. The following theorem is a special case of Theorem 1 in [HT16] by Hilberdink and Tóth .

Theorem 2.11. ([HT16]) Let $f\left(n_{1}, n_{2}\right)=g\left(\left\{n_{1}, n_{2}\right\}\right)$ where $g$ is a multiplicative function satisfying

$$
\sum_{p \in P} \sum_{e \geqq 1} \frac{\left|g\left(p^{e}\right)-g\left(p^{e-1}\right)\right|}{p^{e}}<\infty
$$

Then the mean value $M(f)$ exists and

$$
M(f)=\prod_{p \in P}\left(1+2 \sum_{e \geqq 1} \frac{g\left(p^{e}\right)-g\left(p^{e-1}\right)}{p^{e}}-\sum_{e \geqq 1} \frac{g\left(p^{e}\right)-g\left(p^{e-1}\right)}{p^{2 e}}\right) .
$$

Proof. We prove this theorem using a different method from [HT16]. It is easy to see that for $e, e_{1}, e_{2} \geqq 1$

$$
\begin{aligned}
& (f * \mu)\left(p^{e}, 1\right)=g\left(p^{e}\right)-g\left(p^{e-1}\right), \\
& (f * \mu)\left(p^{e_{1}}, p^{e_{2}}\right)=\left\{\begin{array}{lll}
-\left(g\left(p^{e_{1}}\right)-g\left(p^{e_{1}-1}\right)\right) & \text { if } e_{1}=e_{2} \geqq 1 \\
0 & \text { if } \quad e_{1} \neq e_{2} \text { and } e_{1}, e_{2} \geqq 1 .
\end{array}\right.
\end{aligned}
$$

Since the above relations and (2.26) imply (2.11), Theorem 2.11 holds by Theorem 2.3. This completes the proof of Theorem 2.11.

Now we can prove the following theorem. 
Theorem 2.12. Let $f\left(n_{1}, n_{2}\right)=g\left(\left\{n_{1}, n_{2}\right\}\right)$ where $g$ is a multiplicative function satisfying (2.26). Then its Ramanujan-Fourier series is pointwise convergent and

$$
f\left(n_{1}, n_{2}\right)=\sum_{q_{1}, q_{2}=1}^{\infty} a_{q_{1}, q_{2}} c_{q_{1}}\left(n_{1}\right) c_{q_{2}}\left(n_{2}\right)
$$

holds where

$$
a_{q_{1}, q_{2}}=\sum_{m_{1}, m_{2}=1}^{\infty} \frac{(f * \mu)\left(m_{1} q_{1}, m_{2} q_{2}\right)}{m_{1} q_{1} m_{2} q_{2}} .
$$

Moreover, if $M(f) \neq 0, q, q_{1}, q_{2}>1$ and $\left\{q_{1}, q_{2}\right\}>1$, then $a_{q, 1}, a_{1, q}$ and $a_{q_{1}, q_{2}}$ can be rewritten as follows.

$$
\begin{array}{r}
a_{q, 1}=a_{1, q}=M(f) \prod_{p \mid q} \frac{\sum_{e=\nu_{p}(q)}^{\infty} \frac{g\left(p^{e}\right)-g\left(p^{e-1}\right)}{p^{e}}-\sum_{e=\nu_{p}(q)}^{\infty} \frac{g\left(p^{e}\right)-g\left(p^{e-1}\right)}{p^{2 e}}}{1+2 \sum_{e=1}^{\infty} \frac{g\left(p^{e}\right)-g\left(p^{e-1}\right)}{p^{e}}-\sum_{e=1}^{\infty} \frac{g\left(p^{e}\right)-g\left(p^{e-1}\right)}{p^{2 e}}} \\
a_{q_{1}, q_{2}}=M(f) \prod_{p \mid\left\{q_{1}, q_{2}\right\}} \frac{-\sum_{e=\nu_{p}\left(\left\{q_{1}, q_{2}\right\}\right)}^{\infty} \frac{g\left(p^{e}\right)-g\left(p^{e-1}\right)}{p^{2 e}}}{p^{e}}, \sum_{e=1}^{\infty} \frac{g\left(p^{e}\right)-g\left(p^{e-1}\right)}{p^{\infty}}-\sum_{e=1}^{\infty} \frac{g\left(p^{e}\right)-g\left(p^{e-1}\right)}{p^{2 e}}
\end{array}
$$

Proof. By Theorem 2.4 and Theorem 2.11, we can easily prove this theorem in a similar way as the proof of Theorem 2.7. This completes the proof of Theorem 2.12.

Next we consider a problem such that how $f$ can be determined from given Ramanujan-Fourier coefficients $a_{q_{1}, q_{2}}$. We first consider the case when the Ramanujan-Fourier coefficients $a_{q_{1}, q_{2}}$ is represented in the form $a_{q_{1}, q_{2}}=M(f) h\left(\left(q_{1}, q_{2}\right)\right)$ where $h$ is a given multiplicative function of one variable.

Theorem 2.13. Let $h$ be a multiplicative function of one variable. Then there does not exist a multiplicative function $f: \mathbb{N}^{2} \mapsto \mathbb{C}$ which satisfies the following conditions.

$$
\begin{aligned}
& \sum_{p \in \mathcal{P}} \sum_{\substack{e_{1}, e_{2} \geqq 0 \\
e_{1}+e_{2} \geqq 1}} \frac{\left|(f * \mu)\left(p^{e_{1}}, p^{e_{2}}\right)\right|}{p^{e_{1}+e_{2}}}<\infty, \\
& M(f) \neq 0 \text {, } \\
& f\left(n_{1}, n_{2}\right)=M(f) \sum_{q_{1}, q_{2}=1}^{\infty} h\left(\left(q_{1}, q_{2}\right)\right) c_{q_{1}}\left(n_{1}\right) c_{q_{2}}\left(n_{2}\right) .
\end{aligned}
$$

Proof. Suppose there exists a multiplicative function $f$ which satisfies the above conditions. Then we have from (2.16)

$$
a_{p^{k}, 1} / M(f)=\left(\sum_{e_{1}=k}^{\infty} \sum_{e_{2}=0}^{\infty} \frac{(f * \mu)\left(p^{e_{1}}, p^{e_{2}}\right)}{p^{e_{1}+e_{2}}}\right) /\left(\sum_{e_{1}=0}^{\infty} \sum_{e_{2}=0}^{\infty} \frac{(f * \mu)\left(p^{e_{1}}, p^{e_{2}}\right)}{p^{e_{1}+e_{2}}}\right)=h\left(\left(p^{k}, 1\right)\right)=1
$$

for every $k \geqq 1$. However, this never holds since

$$
\lim _{k \rightarrow \infty} \sum_{e_{1}=k}^{\infty} \sum_{e_{2}=0}^{\infty} \frac{(f * \mu)\left(p^{e_{1}}, p^{e_{2}}\right)}{p^{e_{1}+e_{2}}}=0 .
$$

This completes the proof of Theorem 2.13. 
Next we consider the case when the Ramanujan-Fourier coefficients $a_{q_{1}, q_{2}}$ is represented in the form $a_{q_{1}, q_{2}}=M(f) h\left(q_{1} q_{2}\right)$ where $h$ is a given multiplicative function of one variable.

Theorem 2.14. Let $h$ be a multiplicative function of one variable satisfying $1-2 h(p)+h\left(p^{2}\right) \neq 0$ for every $p \in \mathcal{P}$, and let $f: \mathbb{N}^{2} \mapsto \mathbb{C}$ be a multiplicative function of two variables satisfying

$$
\begin{aligned}
& \sum_{p \in \mathcal{P}} \sum_{\substack{e_{1}, e_{2} \geqq 0 \\
e_{1}+e_{2} \geqq 1}} \frac{\left|(f * \mu)\left(p^{e_{1}}, p^{e_{2}}\right)\right|}{p^{e_{1}+e_{2}}}<\infty, \\
& M(f) \neq 0 \text {, } \\
& f\left(n_{1}, n_{2}\right)=M(f) \sum_{q_{1}, q_{2}=1}^{\infty} h\left(q_{1} q_{2}\right) c_{q_{1}}\left(n_{1}\right) c_{q_{2}}\left(n_{2}\right) .
\end{aligned}
$$

Then $f$ is determined from $h$ as follows.

$$
\begin{aligned}
f\left(p^{k}, 1\right)= & f\left(1, p^{k}\right)=1+\frac{1}{H(p)} \sum_{e=1}^{k} p^{e}(h * \mu * \mu)\left(p^{e+2}\right) \\
f\left(p^{k}, p^{\ell}\right)= & 1+\frac{1}{H(p)} \sum_{e=1}^{k} p^{e}(h * \mu * \mu)\left(p^{e+2}\right)+\frac{1}{H(p)} \sum_{e=1}^{\ell} p^{e}(h * \mu * \mu)\left(p^{e+2}\right) \\
& +\frac{1}{H(p)} \sum_{e_{1}=1}^{k} \sum_{e_{2}=1}^{\ell} p^{e_{1}+e_{2}}(h * \mu * \mu)\left(p^{e_{1}+e_{2}+2}\right)
\end{aligned}
$$

where $H(p)=1-2 h(p)+h\left(p^{2}\right)$ and $k, \ell \geqq 1$.

Proof. We set $F(p)=\sum_{e_{1}=0} \sum_{e_{2}=0} \frac{(f * \mu)\left(p^{e_{1}}, p^{e_{2}}\right)}{p^{e_{1}+e_{2}}}$. By (2.16) we have for any $k, \ell \geqq 0$

$$
\frac{a_{p^{k}, p^{\ell}}}{M(f)}=\frac{1}{F(p)} \sum_{e_{1}=k} \sum_{e_{2}=\ell} \frac{(f * \mu)\left(p^{e_{1}}, p^{e_{2}}\right)}{p^{e_{1}+e_{2}}}=h\left(p^{k+\ell}\right) .
$$

Considering differences of

$$
\sum_{e_{1}=k} \sum_{e_{2}=\ell} \frac{(f * \mu)\left(p^{e_{1}}, p^{e_{2}}\right)}{p^{e_{1}+e_{2}}}=F(p) h\left(p^{k+\ell}\right)
$$

with respect to $k$ and $\ell$, that is, considering

$$
\sum_{e_{1}=k+1} \sum_{e_{2}=\ell+1}-\sum_{e_{1}=k+1} \sum_{e_{2}=\ell}-\sum_{e_{1}=k} \sum_{e_{2}=\ell+1}+\sum_{e_{1}=k} \sum_{e_{2}=\ell}
$$

we have for any $k, \ell \geqq 0$

$$
\begin{aligned}
\frac{(f * \mu)\left(p^{k}, p^{\ell}\right)}{p^{k+\ell}} & =F(p)\left(h\left(p^{k+\ell+2}\right)-2 h\left(p^{k+\ell+1}\right)+h\left(p^{k+\ell}\right)\right) \\
& =F(p)(h * \mu * \mu)\left(p^{k+\ell+2}\right) .
\end{aligned}
$$

Putting $k=\ell=0$ in $(2.30)$ we have $1=F(p)\left(h\left(p^{2}\right)-2 h(p)+1\right)$. From this we obtain

$$
F(p)=\frac{1}{h\left(p^{2}\right)-2 h(p)+1}=\frac{1}{H(p)} .
$$


Putting $\ell=0$ in (2.31) we have for any $k \geqq 1$

$$
\frac{f\left(p^{k}, 1\right)-f\left(p^{k-1}, 1\right)}{p^{k}}=F(p)(h * \mu * \mu)\left(p^{k+2}\right) .
$$

Multiplying both sides by $p^{k}$ and considering summation, we have for any $k \geqq 1$

$$
f\left(p^{k}, 1\right)=1+\frac{1}{H(p)} \sum_{e=1}^{k} p^{e}(h * \mu * \mu)\left(p^{e+2}\right),
$$

which implies (2.28). Next we prove (2.29). We can rewrite (2.31) as follows.

$$
f\left(p^{k}, p^{\ell}\right)-f\left(p^{k}, p^{\ell-1}\right)-f\left(p^{k-1}, p^{\ell}\right)+f\left(p^{k-1}, p^{\ell-1}\right)=\frac{1}{H(p)}(h * \mu * \mu)\left(p^{k+\ell+2}\right) .
$$

Considering summation we have

$$
f\left(p^{k}, p^{\ell}\right)=f\left(p^{k}, 1\right)+f\left(1, p^{\ell}\right)-1+\frac{1}{H(p)} \sum_{e_{1}=1}^{k} \sum_{e_{2}=1}^{\ell} p^{e_{1}+e_{2}}(h * \mu * \mu)\left(p^{e_{1}+e_{2}+2}\right),
$$

which implies (2.29). This completes the proof of Theorem 2.14 .

Next we consider the case when the Ramanujan-Fourier coefficients $a_{q_{1}, q_{2}}$ is represented in the form $a_{q_{1}, q_{2}}=M(f) h\left(\left\{q_{1}, q_{2}\right\}\right)$ where $h$ is a given multiplicative function of one variable.

Theorem 2.15. Let $h$ be a multiplicative function of one variable satisfying

$$
\sum_{e=1}^{\infty}\left|h\left(p^{e}\right)-h\left(p^{e-1}\right)\right|<\infty \quad \text { and } \quad h(p) \neq 1
$$

for every $p \in \mathcal{P}$ and let $f: \mathbb{N}^{2} \mapsto \mathbb{C}$ be a multiplicative function of two variables satisfying

$$
\begin{aligned}
& \sum_{p \in \mathcal{P}} \sum_{\substack{e_{1}, e_{2} \geqq 0 \\
e_{1}+e_{2} \geqq 1}} \frac{\left|(f * \mu)\left(p^{e_{1}}, p^{e_{2}}\right)\right|}{p^{e_{1}+e_{2}}}<\infty, \\
& M(f) \neq 0 \text {, } \\
& f\left(n_{1}, n_{2}\right)=M(f) \sum_{q_{1}, q_{2}=1}^{\infty} h\left(\left\{q_{1}, q_{2}\right\}\right) c_{q_{1}}\left(n_{1}\right) c_{q_{2}}\left(n_{2}\right) .
\end{aligned}
$$

Then there exists a multiplicative function $g: \mathbb{N} \mapsto \mathbb{C}$ such that $f\left(n_{1}, n_{2}\right)=g\left(\left(n_{1}, n_{2}\right)\right)$ for every $n_{1}, n_{2} \in \mathbb{N}$ and

$$
g\left(p^{k}\right)=1-\frac{1}{1-h(p)} \sum_{e=1}^{k} p^{2 e}\left(h\left(p^{e+1}\right)-h\left(p^{e}\right)\right) .
$$

Proof. We set $F(p)=\sum_{e_{1}=0} \sum_{e_{2}=0} \frac{(f * \mu)\left(p^{e_{1}}, p^{e_{2}}\right)}{p^{e_{1}+e_{2}}}$. By (2.16) we have for any $k, \ell \geqq 0$

$$
\frac{a_{p^{k}, p^{\ell}}}{M(f)}=\frac{1}{F(p)} \sum_{e_{1}=k} \sum_{e_{2}=\ell} \frac{(f * \mu)\left(p^{e_{1}}, p^{e_{2}}\right)}{p^{e_{1}+e_{2}}}=h\left(p^{k \vee \ell}\right) .
$$


From this, as in the proof of Theorem 2.14, we have for any $k, \ell \geqq 0$

$$
\begin{aligned}
\frac{(f * \mu)\left(p^{k}, p^{\ell}\right)}{p^{k+\ell}} & =F(p)\left(h\left(p^{(k+1) \vee(\ell+1)}\right)-h\left(p^{k \vee(\ell+1)}\right)-h\left(p^{(k+1) \vee \ell}\right)+h\left(p^{k \vee \ell}\right)\right) \\
& =\left\{\begin{array}{lll}
-F(p)\left(h\left(p^{k+1}\right)-h\left(p^{k}\right)\right) & \text { if } & k=\ell \geqq 0 \\
0 & \text { if } & k \neq \ell .
\end{array}\right.
\end{aligned}
$$

Putting $k=\ell=0$ in $(2.34)$ we have $1=-F(p)(h(p)-1)$. From this we obtain

$$
F(p)=\frac{1}{1-h(p)} \text {. }
$$

Putting $\ell=0$ in (2.34) we have for any $k \geqq 1$

$$
\frac{f\left(p^{k}, 1\right)-f\left(p^{k-1}, 1\right)}{p^{k}}=0 .
$$

From this it follows that for any $k \geqq 1$

$$
f\left(p^{k}, 1\right)=f(1,1)=1 .
$$

When $k>\ell \geqq 1$, we can rewrite (2.34) as follows.

$$
f\left(p^{k}, p^{\ell}\right)-f\left(p^{k-1}, p^{\ell}\right)-f\left(p^{k}, p^{\ell-1}\right)+f\left(p^{k-1}, p^{\ell-1}\right)=0 .
$$

From this and (2.35) we have

$$
f\left(p^{k}, p^{\ell}\right)-f\left(p^{k-1}, p^{\ell}\right)=f\left(p^{k}, p^{\ell-1}\right)-f\left(p^{k-1}, p^{\ell-1}\right)=\cdots=f\left(p^{k}, 1\right)-f\left(p^{k-1}, 1\right)=0,
$$

and

$$
f\left(p^{k}, p^{\ell}\right)=f\left(p^{k-1}, p^{\ell}\right)=\cdots=f\left(p^{\ell}, p^{\ell}\right)=f\left(p^{k \wedge \ell}, p^{k \wedge \ell}\right) .
$$

Therefore, if we define the multiplicative function $g: \mathbb{N} \mapsto \mathbb{C}$ by the relation $g\left(p^{e}\right)=f\left(p^{e}, p^{e}\right)$ for $e \geqq 0$, then we have

$$
f\left(p^{k}, p^{\ell}\right)=f\left(p^{k \wedge \ell}, p^{k \wedge \ell}\right)=g\left(p^{k \wedge \ell}\right)=g\left(\left(p^{k}, p^{\ell}\right)\right) .
$$

Since $g$ is defined to be multiplicative, $f\left(n_{1}, n_{2}\right)=g\left(\left(n_{1}, n_{2}\right)\right)$ holds for every $n_{1}, n_{2} \in \mathbb{N}$.

In order to show (2.33), we put $k=\ell \geqq 1$ in (2.34) and obtain

$$
\frac{f\left(p^{k}, p^{k}\right)-f\left(p^{k}, p^{k-1}\right)-f\left(p^{k-1}, p^{k}\right)+f\left(p^{k-1}, p^{k-1}\right)}{p^{2 k}}=-F(p)\left(h\left(p^{k+1}\right)-h\left(p^{k}\right)\right) .
$$

From this we have for any $k \geqq 1$

$$
g\left(p^{k}\right)-g\left(p^{k-1}\right)=-\frac{1}{1-h(p)} p^{2 k}\left(h\left(p^{k+1}\right)-h\left(p^{k}\right)\right) .
$$

Considering summation, we see that (2.33) holds. This completes the proof of Theorem 2.15.

\section{Examples}

In this section, we give several examples. We begin with the following example which is a special case of Theorem 2.7 . 
Example 3.1. Let $f\left(n_{1}, n_{2}\right)=\varphi\left(n_{1} n_{2}\right) / n_{1} n_{2}$. Then its Ramanujan-Fourier series is pointwise convergent and

$$
\frac{\varphi\left(n_{1} n_{2}\right)}{n_{1} n_{2}}=M(f) \sum_{q_{1}, q_{2}=1}^{\infty} \frac{\mu\left(q_{1}\right) \mu\left(q_{2}\right)}{\varphi\left(\left(q_{1}, q_{2}\right)\right) \tilde{\varphi}\left(q_{1} q_{2}\right)} c_{q_{1}}\left(n_{1}\right) c_{q_{2}}\left(n_{2}\right)
$$

holds where $M(f)=\prod_{p \in \mathcal{P}}\left(1-2 / p^{2}+1 / p^{3}\right)$ and $\tilde{\varphi}(n)=\prod_{p \mid n}\left(p^{2}+p-1\right)$.

Proof. Setting $g(n)=\varphi(n) / n=\prod_{p \mid n}(1-1 / p)$, we have

$$
\begin{aligned}
& g\left(p^{e}\right)=1-1 / p \quad \text { if } \quad e \geqq 1 \\
& g\left(p^{e}\right)-g\left(p^{e-1}\right)=\left\{\begin{array}{cll}
-1 / p & \text { if } & e=1 \\
0 & \text { if } & e \geqq 2,
\end{array}\right. \\
& g\left(p^{e}\right)-2 g\left(p^{e-1}\right)+g\left(p^{e-2}\right)=\left\{\begin{array}{cll}
1 / p & \text { if } & e=2 \\
0 & \text { if } & e \geqq 3
\end{array}\right.
\end{aligned}
$$

Therefore $g$ clearly satisfies (2.17). Using (2.18) we can calculate the mean value $M(f)$ as follows.

$$
M(f)=\prod_{p \in \mathcal{P}}\left(1+2 \frac{-1 / p}{p}+\frac{1 / p}{p^{2}}\right)=\prod_{p \in \mathcal{P}}\left(1-\frac{2}{p^{2}}+\frac{1}{p^{3}}\right) .
$$

Since $M(f) \neq 0$, we have by Theorem 2.7

$$
\begin{aligned}
& a_{p, 1}=M(f)\left(-\frac{1}{p^{2}}+\frac{1}{p^{3}}\right) /\left(1-\frac{2}{p^{2}}+\frac{1}{p^{3}}\right)=-\frac{M(f)}{p^{2}+p-1}, \\
& a_{p^{k}, 1}=0 \text { if } k \geqq 2, \\
& a_{p, p}=M(f)\left(\frac{1}{p^{3}}\right) /\left(1-\frac{2}{p^{2}}+\frac{1}{p^{3}}\right)=\frac{M(f)}{(p-1)\left(p^{2}+p-1\right)}, \\
& a_{p^{k}, p^{\ell}}=0 \text { if } k, \ell \geqq 1 \text { and } k+\ell \geqq 3 .
\end{aligned}
$$

Hence

$$
a_{q_{1}, q_{2}}=M(f) \frac{\mu\left(q_{1}\right) \mu\left(q_{2}\right)}{\varphi\left(\left(q_{1}, q_{2}\right)\right) \tilde{\varphi}\left(q_{1} q_{2}\right)}
$$

holds if $\left(q_{1}, q_{2}\right)=\left(p^{k}, p^{\ell}\right)$ where $k, \ell \geqq 0$. Since the function $\left(q_{1}, q_{2}\right) \mapsto a_{q_{1}, q_{2}} / M(f)$ is multiplicative, (3.36) holds for every $q_{1}, q_{2} \in \mathbb{N}$. This completes the proof of Example 3.1.

The formula (1.3) says that, if we set $f(n)=\varphi(n) / n$, then $f(n)=M(f) \sum_{q=1}^{\infty}\left(\mu(q) / \varphi_{2}(q)\right) c_{q}(n)$ holds since $M(f)=6 / \pi^{2}$ in this case. However, Example 3.1 shows that the function $f\left(n_{1}, n_{2}\right)=$ $\varphi\left(n_{1} n_{2}\right) / n_{1} n_{2}$ does not have a similar expression of Ramanujan-Fourier series to that of (1.3). We want to find an arithmetic function $f$ satisfying $f\left(n_{1}, n_{2}\right)=M(f) \sum_{q_{1}, q_{2}=1}^{\infty} \frac{\mu\left(q_{1} q_{2}\right)}{\varphi_{2}\left(q_{1} q_{2}\right)} c_{q_{1}}\left(n_{1}\right) c_{q_{2}}\left(n_{2}\right)$. In the following example, we give a function which satisfies the above relation.

Example 3.2. Let $f\left(n_{1}, n_{2}\right)$ be the multiplicative function defined by

$$
f\left(p^{k}, p^{\ell}\right)= \begin{cases}1-p /\left(p^{2}+1\right) & \text { if } \quad k \text { or } \ell=0 \text { and } k+\ell \geqq 1 \\ 1-2 p /\left(p^{2}+1\right) & \text { if } \quad k, \ell \geqq 1 .\end{cases}
$$

Then its Ramanujan-Fourier series is pointwise convergent and

$$
f\left(n_{1}, n_{2}\right)=M(f) \sum_{q_{1}, q_{2}=1}^{\infty} \frac{\mu\left(q_{1} q_{2}\right)}{\varphi_{2}\left(q_{1} q_{2}\right)} c_{q_{1}}\left(n_{1}\right) c_{q_{2}}\left(n_{2}\right)
$$

holds where

$$
M(f)=\prod_{p \in \mathcal{P}}\left(1-\frac{2}{p^{2}+1}\right) .
$$


Proof. By (3.37) it is easy to see that

$$
(f * \mu)\left(p^{k}, p^{\ell}\right)= \begin{cases}-p /\left(p^{2}+1\right) & \text { if }(k, \ell)=(1,0) \text { or }(0,1) \\ 0 & \text { otherwise. }\end{cases}
$$

From the above relation it is obvious that $f$ satisfies (2.13). Hence its Ramanujan-Fourier series is pointwise convergent by Theorem 2.4. Using (2.12) and (3.38) we have

$$
M(f)=\prod_{p \in \mathcal{P}}\left(1+\frac{(f * \mu)(p, 1)+(f * \mu)(1, p)}{p}\right)=\prod_{p \in \mathcal{P}}\left(1-\frac{2}{p^{2}+1}\right) .
$$

As for the Ramanujan-Fourier coefficients of $f$, we obtain by (2.16) and (3.38)

$$
\begin{aligned}
& a_{p^{k}, 1}=a_{1, p^{k}}= \begin{cases}M(f)\left(-\frac{1}{p^{2}+1}\right) /\left(1-\frac{2}{p^{2}+1}\right)=M(f) \frac{-1}{p^{2}-1}=M(f) \frac{\mu(p)}{\varphi_{2}(p)} & \text { if } \quad k=1 \\
0=M(f) \frac{\mu\left(p^{k}\right)}{\varphi_{2}\left(p^{k}\right)} & \text { if } \quad k \geqq 2,\end{cases} \\
& a_{p^{k}, p^{\ell}}=0=M(f) \frac{\mu\left(p^{k} p^{\ell}\right)}{\varphi_{2}\left(p^{k} p^{\ell}\right)} \text { if } k, \ell \geqq 1 .
\end{aligned}
$$

Hence

$$
a_{q_{1}, q_{2}}=M(f) \frac{\mu\left(q_{1} q_{2}\right)}{\varphi_{2}\left(q_{1} q_{2}\right)}
$$

holds if $\left(q_{1}, q_{2}\right)=\left(p^{k}, p^{\ell}\right)$ where $k, \ell \geqq 0$. Since the function $\left(q_{1}, q_{2}\right) \mapsto a_{q_{1}, q_{2}} / M(f)$ is multiplicative, (3.39) holds for every $q_{1}, q_{2} \in \mathbb{N}$. This completes the proof of Example 3.2.

Remark 3.3. If we put $h(n)=\mu(n) / \varphi_{2}(n)$ in Theorem 2.14, then it is easy to see that $f$ satisfies (3.37) by (2.28) and (2.29). Thus we can make use of (2.28) and (2.29) when we want to find the explicit function form of $f$ satisfying $f\left(n_{1}, n_{2}\right)=M(f) \sum_{q_{1}, q_{2}=1}^{\infty} h\left(n_{1} n_{2}\right) c_{q_{1}}\left(n_{1}\right) c_{q_{2}}\left(n_{2}\right)$ for given $h$.

The following example is similar to Example 3.2.

Example 3.4. Let $f\left(n_{1}, n_{2}\right)$ be the multiplicative function defined by

$$
f\left(p^{k}, p^{\ell}\right)= \begin{cases}1-p /\left(p^{s}+2\right) & \text { if } \quad k \text { or } \ell=0 \text { and } k+\ell \geqq 1 \\ 1-2 p /\left(p^{s}+2\right) & \text { if } k, \ell \geqq 1,\end{cases}
$$

where $s>1$. Then its Ramanujan-Fourier series is pointwise convergent and

$$
f\left(n_{1}, n_{2}\right)=M(f) \sum_{q_{1}, q_{2}=1}^{\infty} \frac{\mu\left(q_{1} q_{2}\right)}{\left(q_{1} q_{2}\right)^{s}} c_{q_{1}}\left(n_{1}\right) c_{q_{2}}\left(n_{2}\right)
$$

holds where

$$
M(f)=\prod_{p \in \mathcal{P}}\left(1+2 / p^{s}\right)^{-1} .
$$

Proof. The proof is similar to that of Example 3.2. We note that

$$
(f * \mu)\left(p^{k}, p^{\ell}\right)= \begin{cases}-p /\left(p^{s}+2\right) & \text { if }(k, \ell)=(1,0) \text { or }(0,1) \\ 0 & \text { otherwise. }\end{cases}
$$

From this we have

$$
M(f)=\prod_{p \in \mathcal{P}}\left(1+\frac{(f * \mu)(p, 1)+(f * \mu)(1, p)}{p}\right)=\prod_{p \in \mathcal{P}}\left(1-\frac{2}{p^{s}+2}\right)=\prod_{p \in \mathcal{P}} \frac{1}{1+2 / p^{s}} .
$$


As for the Ramanujan-Fourier coefficients of $f$, we obtain by (2.16) and (3.41)

$$
\begin{aligned}
& a_{p^{k}, 1}=a_{1, p^{k}}= \begin{cases}M(f)\left(-\frac{1}{p^{s}+2}\right) /\left(1-\frac{2}{p^{s}+2}\right)=M(f) \frac{-1}{p^{s}}=M(f) \frac{\mu(p)}{p^{s}} & \text { if } \quad k=1 \\
0=M(f) \frac{\mu\left(p^{k}\right)}{\left(p^{k}\right)^{s}} & \text { if } \quad k \geqq 2,\end{cases} \\
& a_{p^{k}, p^{\ell}}=0=M(f) \frac{\mu\left(p^{k} p^{\ell}\right)}{\left(p^{k} p^{\ell}\right)^{s}} \text { if } k, \ell \geqq 1 .
\end{aligned}
$$

Hence

$$
a_{q_{1}, q_{2}}=M(f) \frac{\mu\left(q_{1} q_{2}\right)}{\left(q_{1} q_{2}\right)^{s}}
$$

holds if $\left(q_{1}, q_{2}\right)=\left(p^{k}, p^{\ell}\right)$ where $k, \ell \geqq 0$. Since the function $\left(q_{1}, q_{2}\right) \mapsto a_{q_{1}, q_{2}} / M(f)$ is multiplicative, (3.42) holds for every $q_{1}, q_{2} \in \mathbb{N}$. This completes the proof of Example 3.4.

Remark 3.5. If we put $h(n)=\mu(n) / n^{s}$ in Theorem 2.14, then it is easy to see that $f$ satisfies (3.40) by (2.28) and (2.29).

The following two examples are special cases of Theorem 2.7.

Example 3.6. Let $f\left(n_{1}, n_{2}\right)=\mu^{2}\left(n_{1} n_{2}\right)$. Then its Ramanujan-Fourier series is pointwise convergent and

$$
\mu^{2}\left(n_{1} n_{2}\right)=M(f) \sum_{q_{1}, q_{2}=1}^{\infty} a_{q_{1}, q_{2}} c_{q_{1}}\left(n_{1}\right) c_{q_{2}}\left(n_{2}\right),
$$

where $M(f)=\prod_{p \in \mathcal{P}}\left(1-3 / p^{2}+2 / p^{3}\right)$, and $a_{q_{1}, q_{2}}$ is determined by the multiplicativity of $\left(q_{1}, q_{2}\right) \mapsto$ $a_{q_{1}, q_{2}} / M(f)$ and the following relations.

$$
a_{p^{k}, p^{\ell}} / M(f)= \begin{cases}1 & \text { if } \quad(k, \ell)=(0,0) \\ -2 /\left(p^{2}+p-2\right) & \text { if }(k, \ell)=(1,0) \text { or }(0,1) \\ -1 /\left(p^{2}+p-2\right) & \text { if }(k, \ell)=(2,0) \text { or } \quad(0,2) \\ (-p+2) /\left(p^{3}-3 p+2\right) & \text { if }(k, \ell)=(1,1) \\ 1 /\left(p^{3}-3 p+2\right) & \text { if }(k, \ell)=(2,1) \text { or }(1,2) \\ 0 & \text { otherwise. }\end{cases}
$$

Proof. We proceed as in the proof of Example 3.1. If we set $g(n)=\mu^{2}(n)$, then we have

$$
\begin{aligned}
& g\left(p^{e}\right)= \begin{cases}1 & \text { if } \quad e=0 \text { or } 1 \\
0 & \text { if } e \geqq 2\end{cases} \\
& g\left(p^{e}\right)-g\left(p^{e-1}\right)=\left\{\begin{array}{rll}
-1 & \text { if } & e=2 \\
0 & \text { if } & e=1 \quad \text { or } e \geqq 3
\end{array}\right. \\
& g\left(p^{e}\right)-2 g\left(p^{e-1}\right)+g\left(p^{e-2}\right)=\left\{\begin{array}{rll}
-1 & \text { if } & e=2 \\
1 & \text { if } & e=3 \\
0 & \text { if } & e \geqq 4
\end{array}\right.
\end{aligned}
$$

Therefore $g$ clearly satisfies (2.17). Using (2.18) we can calculate the mean value $M(f)$ as follows.

$$
M(f)=\prod_{p \in \mathcal{P}}\left(1+2 \times \frac{-1}{p^{2}}+\left(\frac{-1}{p^{2}}+\frac{2}{p^{3}}\right)\right)=\prod_{p \in \mathcal{P}}\left(1-\frac{3}{p^{2}}+\frac{2}{p^{3}}\right) .
$$


As for the Ramanujan-Fourier coefficients $a_{q_{1}, q_{2}}$, we have by Theorem 2.7

$$
\begin{aligned}
& a_{p, 1}=M(f)\left(-\frac{1}{p^{2}}+\left(-\frac{1}{p^{2}}+\frac{1+1}{p^{3}}\right)\right) /\left(1-\frac{3}{p^{2}}+\frac{2}{p^{3}}\right)=M(f) \frac{-2}{p^{2}+p-2}, \\
& a_{p^{2}, 1}=M(f)\left(-\frac{1}{p^{2}}+\frac{1}{p^{3}}\right) /\left(1-\frac{3}{p^{2}}+\frac{2}{p^{3}}\right)=M(f) \frac{-1}{p^{2}+p-2}, \\
& a_{p^{k}, 1}=0 \text { if } k \geqq 3, \\
& a_{p, p}=M(f)\left(\frac{-1}{p^{2}}+\frac{2}{p^{3}}\right) /\left(1-\frac{3}{p^{2}}+\frac{2}{p^{3}}\right)=M(f) \frac{-p+2}{p^{3}-3 p+2}, \\
& a_{p^{2}, p}=M(f)\left(\frac{1}{p^{3}}\right) /\left(1-\frac{3}{p^{2}}+\frac{2}{p^{3}}\right)=M(f) \frac{1}{p^{3}-3 p+2}, \\
& a_{p^{k}, p^{\ell}}=0 \text { if } k \vee \ell \geqq 3 \text { or } k+\ell \geqq 4 .
\end{aligned}
$$

This completes the proof of Example 3.6.

The following example is an extension of (1.2) to the case $f\left(n_{1}, n_{2}\right)=g\left(n_{1} n_{2}\right)$.

Example 3.7. Let $f\left(n_{1}, n_{2}\right)=\sigma_{s}\left(n_{1} n_{2}\right) /\left(n_{1} n_{2}\right)^{s}$ where $s>0$. Then its Ramanujan-Fourier series is pointwise convergent and

$$
\frac{\sigma_{s}\left(n_{1} n_{2}\right)}{\left(n_{1} n_{2}\right)^{s}}=M(f) \sum_{q_{1}, q_{2}=1}^{\infty} a_{q_{1}, q_{2}} c_{q_{1}}\left(n_{1}\right) c_{q_{2}}\left(n_{2}\right),
$$

where $M(f)=\zeta^{2}(s+1) / \zeta(s+2)$, and $a_{q_{1}, q_{2}}$ is determined by the multiplicativity of $\left(q_{1}, q_{2}\right) \mapsto$ $a_{q_{1}, q_{2}} / M(f)$ and the following relations.

$$
\begin{aligned}
& a_{p^{k}, 1} / M(f)=a_{1, p^{k}} / M(f)=\frac{1-1 / p}{p^{k(s+1)}\left(1-1 / p^{s+2}\right)}, \\
& a_{p^{k}, p^{\ell}} / M(f)=\frac{1-p^{s}}{p^{(k+\ell)(s+1)}\left(1-1 / p^{s+2}\right)} .
\end{aligned}
$$

Proof. If we set $g(n)=\sigma_{s}(n) / n^{s}$, then we have

$$
\begin{aligned}
g\left(p^{e}\right) & =\frac{1}{p^{e s}} \frac{1-p^{(e+1) s}}{1-p^{s}}=\frac{1}{1-p^{s}}\left(\frac{1}{p^{e s}}-p^{s}\right) \quad \text { if } \quad e \geqq 0 \\
g\left(p^{e}\right)-g\left(p^{e-1}\right) & =\frac{1}{1-p^{s}}\left(\frac{1}{p^{e s}}-\frac{1}{p^{(e-1) s}}\right)=\frac{1}{1-p^{s}} \frac{1-p^{s}}{p^{e s}}=\frac{1}{p^{e s}} \quad \text { if } \quad e \geqq 1 \\
g\left(p^{e}\right)-2 g\left(p^{e-1}\right)+g\left(p^{e-2}\right) & =\frac{1}{p^{e s}}-\frac{1}{p^{(e-1) s}}=\frac{1-p^{s}}{p^{e s}} \quad \text { if } \quad e \geqq 2 .
\end{aligned}
$$

Therefore $g$ clearly satisfies (2.17). Using (2.18) we can calculate the mean value $M(f)$ as follows

$$
\begin{aligned}
M(f) & =\prod_{p \in \mathcal{P}}\left(1+2 \sum_{e \geqq 1} \frac{1}{p^{e}} \frac{1}{p^{e s}}+\sum_{e_{1} \geqq 1} \sum_{e_{2} \geqq 1} \frac{1}{p^{e_{1}+e_{2}}} \frac{1-p^{s}}{p^{\left(e_{1}+e_{2}\right) s}}\right) \\
& =\prod_{p \in \mathcal{P}} \frac{1-1 / p^{s+2}}{\left(1-1 / p^{s+1}\right)^{2}}=\frac{\zeta^{2}(s+1)}{\zeta(s+2)} .
\end{aligned}
$$


As for the Ramanujan-Fourier coefficients $a_{q_{1}, q_{2}}$, we have by Theorem 2.7

$$
\begin{aligned}
a_{p^{k}, 1} & =M(f)\left(\sum_{e=k}^{\infty} \frac{1}{p^{e}} \frac{1}{p^{e s}}+\sum_{e_{1}=k}^{\infty} \sum_{e_{2}=1}^{\infty} \frac{1}{p^{e_{1}+e_{2}}} \frac{1-p^{s}}{p^{\left(e_{1}+e_{2}\right) s}}\right) \frac{\left(1-1 / p^{s+1}\right)^{2}}{1-1 / p^{s+2}} \\
& =M(f) \frac{1-1 / p}{p^{k(s+1)}\left(1-1 / p^{s+2}\right)}, \\
a_{p^{k}, p^{\ell}} & =M(f)\left(\sum_{e_{1}=k}^{\infty} \sum_{e_{2}=\ell}^{\infty} \frac{1}{p^{e_{1}+e_{2}}} \frac{1-p^{s}}{p^{\left(e_{1}+e_{2}\right) s}}\right) \frac{\left(1-1 / p^{s+1}\right)^{2}}{1-1 / p^{s+2}} \\
& =M(f) \frac{1-p^{s}}{p^{(k+\ell)(s+1)}\left(1-1 / p^{s+2}\right)} .
\end{aligned}
$$

This completes the proof of Example 3.7.

Next we give examples which are special cases of Theorem 2.10. The following example is an extension of (1.2) to the case $f\left(n_{1}, n_{2}\right)=g\left(\left(n_{1}, n_{2}\right)\right)$.

Example 3.8. Let $f\left(n_{1}, n_{2}\right)=\sigma_{s}\left(\left(n_{1}, n_{2}\right)\right) /\left(n_{1}, n_{2}\right)^{\text {s }}$ where $s>-1$. Then its Ramanujan-Fourier series is pointwise convergent and

$$
\frac{\sigma_{s}\left(\left(n_{1}, n_{2}\right)\right)}{\left(n_{1}, n_{2}\right)^{s}}=\zeta(s+2) \sum_{q_{1}, q_{2}=1}^{\infty} \frac{c_{q_{1}}\left(n_{1}\right) c_{q_{2}}\left(n_{2}\right)}{\left\{q_{1}, q_{2}\right\}^{s+2}} .
$$

Proof. As in the proof of Example 3.7, if we set $g(n)=\sigma_{s}(n) / n^{s}$, then we have

$$
\begin{aligned}
& g\left(p^{e}\right)=\frac{1}{1-p^{s}}\left(\frac{1}{p^{e s}}-p^{s}\right) \text { for } e \geqq 0, \\
& g\left(p^{e}\right)-g\left(p^{e-1}\right)=\frac{1}{p^{e s}} \text { for } e \geqq 1 .
\end{aligned}
$$

Therefore (2.21) holds and we have by $(2.22)$

$$
M(f)=\prod_{p \in \mathcal{P}}\left(1+\sum_{e=1}^{\infty} \frac{1}{p^{(s+2) e}}\right)=\zeta(s+2) .
$$

For $k$ and $\ell$ satisfying $k+\ell \geqq 1$ we also have by $(2.25)$

$$
\begin{aligned}
a_{p^{k}, p^{\ell}} & =M(f) \frac{\sum_{e=k \vee \ell}^{\infty} \frac{g\left(p^{e}\right)-g\left(p^{e-1}\right)}{p^{2 e}}}{1+\sum_{e=1}^{\infty} \frac{g\left(p^{e}\right)-g\left(p^{e-1}\right)}{p^{2 e}}}=M(f) \frac{\sum_{e=k \vee \ell}^{\infty} \frac{1}{p^{(2+s) e}}}{1+\sum_{e=1}^{\infty} \frac{1}{p^{(2+s) e}}} \\
& =M(f) \frac{\frac{1}{p^{(2+s)(k \vee \ell)} \frac{1}{1-\frac{1}{p^{2+s}}}}}{\frac{1}{1-\frac{1}{p^{2+s}}}}=M(f) \frac{1}{p^{(2+s)(k \vee \ell)}}=M(f) \frac{1}{\left\{p^{k}, p^{\ell}\right\}^{2+s}} .
\end{aligned}
$$

Therefore $a_{q_{1}, q_{2}}=M(f) /\left\{p^{k}, p^{\ell}\right\}^{2+s}$ holds if $\left(q_{1}, q_{2}\right)=\left(p^{k}, p^{\ell}\right)$ where $k, \ell \geqq 0$. Since the function $\left(q_{1}, q_{2}\right) \mapsto a_{q_{1}, q_{2}} / M(f)$ is multiplicative, we obtain the desired result. This completes the proof of Example 3.8.

If we set $s=0$ in Example 3.8, then we obtain the following example.

Example 3.9. Let $f\left(n_{1}, n_{2}\right)=\tau\left(\left(n_{1}, n_{2}\right)\right)$ where $\tau(n)$ is the number of divisors of $n$. Then its Ramanujan-Fourier series is pointwise convergent and

$$
\tau\left(\left(n_{1}, n_{2}\right)\right)=\zeta(2) \sum_{q_{1}, q_{2}=1}^{\infty} \frac{c_{q_{1}}\left(n_{1}\right) c_{q_{2}}\left(n_{2}\right)}{\left\{q_{1}, q_{2}\right\}^{2}} .
$$


The following example is an extension of (1.3) to the case $f\left(n_{1}, n_{2}\right)=g\left(\left(n_{1}, n_{2}\right)\right)$.

Example 3.10. Let $f\left(n_{1}, n_{2}\right)=\varphi\left(\left(n_{1}, n_{2}\right)\right) /\left(n_{1}, n_{2}\right)$. Then its Ramanujan-Fourier series is pointwise convergent and

$$
\frac{\varphi\left(\left(n_{1}, n_{2}\right)\right)}{\left(n_{1}, n_{2}\right)}=\frac{1}{\zeta(3)} \sum_{q_{1}, q_{2}=1}^{\infty} \frac{\mu\left(\left\{q_{1}, q_{2}\right\}\right)}{\varphi_{3}\left(\left\{q_{1}, q_{2}\right\}\right)} c_{q_{1}}\left(n_{1}\right) c_{q_{2}}\left(n_{2}\right) .
$$

Proof. If we set $g(n)=\varphi(n) / n$, then $g\left(p^{e}\right)=1-1 / p$ for $e \geqq 1$. Hence (2.21) holds and we have by $(2.22)$

$$
M(f)=\prod_{p \in \mathcal{P}}\left(1+\sum_{e=1}^{\infty} \frac{g\left(p^{e}\right)-g\left(p^{e-1}\right)}{p^{2 e}}\right)=\prod_{p \in \mathcal{P}}\left(1+\frac{(1-1 / p)-1}{p^{2}}\right)=\prod_{p \in \mathcal{P}}\left(1-\frac{1}{p^{3}}\right)=\frac{1}{\zeta(3)} .
$$

We also have by $(2.25)$

$$
a_{p^{k}, p^{\ell}}=M(f) \frac{\sum_{e=k \vee \ell}^{\infty} \frac{g\left(p^{e}\right)-g\left(p^{e-1}\right)}{p^{2 e}}}{1+\sum_{e=1}^{\infty} \frac{g\left(p^{e}\right)-g\left(p^{e-1}\right)}{p^{2 e}}}=0 \text { if } k \vee \ell \geqq 2
$$

and

$$
a_{p, 1}=a_{1, p}=a_{p, p}=M(f) \frac{-1 / p^{3}}{1-1 / p^{3}}=M(f) \frac{-1}{p^{3}\left(1-1 / p^{3}\right)} .
$$

Therefore $a_{q_{1}, q_{2}}=M(f) \mu\left(\left\{q_{1}, q_{2}\right\}\right) / \varphi_{3}\left(\left\{q_{1}, q_{2}\right\}\right)$ holds if $\left(q_{1}, q_{2}\right)=\left(p^{k}, p^{\ell}\right)$ where $k, \ell \geqq 0$. Since the function $\left(q_{1}, q_{2}\right) \mapsto a_{q_{1}, q_{2}} / M(f)$ is multiplicative, we obtain the desired result.

The proof of the following example is similar to that of the previous example.

Example 3.11. Let $f\left(n_{1}, n_{2}\right)=\varphi_{s}\left(\left(n_{1}, n_{2}\right)\right) /\left(n_{1}, n_{2}\right)^{s}$ where $s>-1$. Then its Ramanujan-Fourier series is pointwise convergent and

$$
\frac{\varphi_{s}\left(\left(n_{1}, n_{2}\right)\right)}{\left(n_{1}, n_{2}\right)^{s}}=\frac{1}{\zeta(s+2)} \sum_{q_{1}, q_{2}=1}^{\infty} \frac{\mu\left(\left\{q_{1}, q_{2}\right\}\right)}{\varphi_{s+2}\left(\left\{q_{1}, q_{2}\right\}\right)} c_{q_{1}}\left(n_{1}\right) c_{q_{2}}\left(n_{2}\right) .
$$

If we set $s=0$ in Example 3.11, then we obtain the following example.

Example 3.12. Let $f\left(n_{1}, n_{2}\right)=\delta\left(\left(n_{1}, n_{2}\right)\right)=\left\{\begin{array}{lll}1 & \text { if } & \left(n_{1}, n_{2}\right)=1 \\ 0 & \text { if } & \left(n_{1}, n_{2}\right)>1 .\end{array} \quad\right.$ Then its Ramanujan-Fourier series is pointwise convergent and

$$
\delta\left(\left(n_{1}, n_{2}\right)\right)=\frac{1}{\zeta(2)} \sum_{q_{1}, q_{2}=1}^{\infty} \frac{\mu\left(\left\{q_{1}, q_{2}\right\}\right)}{\varphi_{2}\left(\left\{q_{1}, q_{2}\right\}\right)} c_{q_{1}}\left(n_{1}\right) c_{q_{2}}\left(n_{2}\right) .
$$

The following example is an extension of (1.5) to the case $f\left(n_{1}, n_{2}\right)=g\left(\left(n_{1}, n_{2}\right)\right)$.

Example 3.13. Let $f\left(n_{1}, n_{2}\right)=\frac{1}{4} r\left(\left(n_{1}, n_{2}\right)\right)$ where $r(n)=\#\left\{(A, B) \in \mathbb{Z} \times \mathbb{Z} ; A^{2}+B^{2}=n\right\}$. Then its Ramanujan-Fourier series is pointwise convergent and

$$
\frac{1}{4} r\left(\left(n_{1}, n_{2}\right)\right)=M(f) \sum_{q_{1}, q_{2}=1}^{\infty} \frac{\chi\left(\left\{q_{1}, q_{2}\right\}\right)}{\left\{q_{1}, q_{2}\right\}^{2}} c_{q_{1}}\left(n_{1}\right) c_{q_{2}}\left(n_{2}\right),
$$

where $\chi(n)=\left\{\begin{array}{ccc}0 & \text { if } n \text { is even } \\ (-1)^{\frac{n-1}{2}} & \text { if } n \text { is odd }\end{array}\right.$ and $M(f)=\prod_{p>2, p \in \mathcal{P}} \frac{1}{1-\chi(p) / p^{2}}$. 
Proof. Let $g(n)=\frac{1}{4} r(n)$ and $e \in \mathbb{N}$. Then $g\left(2^{e}\right)=1$ and

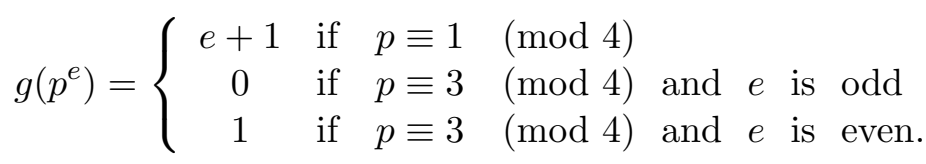

From this we have $g\left(2^{e}\right)-g\left(2^{e-1}\right)=0$ and

$$
g\left(p^{e}\right)-g\left(p^{e-1}\right)=\left\{\begin{array}{rllll}
1 & \text { if } & p \equiv 1 & (\bmod 4) & \\
-1 & \text { if } & p \equiv 3 & (\bmod 4) & \text { and } e \text { is odd } \\
1 & \text { if } & p \equiv 3 & (\bmod 4) & \text { and } e \text { is even. }
\end{array}\right.
$$

Hence (2.21) clearly holds. Furthermore, we have for $k \geqq 1$

$$
\sum_{e=k}^{\infty} \frac{g\left(p^{e}\right)-g\left(p^{e-1}\right)}{p^{2 e}}=\left\{\begin{array}{lll}
0 & \text { if } p=2 \\
\sum_{e=k}^{\infty} \frac{1}{p^{2 e}}=\frac{1}{p^{2 k}} \frac{1}{1-1 / p^{2}} & \text { if } p \equiv 1 \quad(\bmod 4) \\
\sum_{e=k}^{\infty} \frac{(-1)^{2}}{p^{2 e}}=-\frac{1}{p^{2 k}} \frac{1}{1+1 / p^{2}} & \text { if } p \equiv 3 \quad(\bmod 4) \text { and } k \text { is odd } \\
\sum_{e=k}^{\infty} \frac{(-1)^{e}}{p^{2 e}}=\frac{1}{p^{2 k}} \frac{1}{1+1 / p^{2}} & \text { if } p \equiv 3 \quad(\bmod 4) \text { and } k \text { is even. }
\end{array}\right.
$$

Using (2.22) and(3.43) we can calculate the mean value $M(f)$ as follows.

$$
\begin{aligned}
M(f) & =\prod_{p \equiv 1(\bmod 4)}\left(1+\frac{1}{p^{2}\left(1-1 / p^{2}\right)}\right) \prod_{p \equiv 3(\bmod 4)}\left(1-\frac{1}{p^{2}\left(1+1 / p^{2}\right)}\right) \\
& =\prod_{p \equiv 1(\bmod 4)} \frac{1}{1-1 / p^{2}} \prod_{p \equiv 3(\bmod 4)} \frac{1}{1+1 / p^{2}}=\prod_{p>2, p \in \mathcal{P}} \frac{1}{1-\chi(p) / p^{2}} .
\end{aligned}
$$

Let $k, \ell$ be non-negative integers satisfying $k+\ell \geqq 1$. Using (2.25) and (3.43) we obtain

$$
\begin{aligned}
& a_{2^{k}, 2^{\ell}}=0 \\
& a_{p^{k}, p^{\ell}}=M(f) \frac{\frac{1}{p^{2(k \vee \ell)} \frac{1}{1-1 / p^{2}}}}{1+\frac{1}{p^{2}-1}}=M(f) \frac{1}{p^{2(k \vee \ell)}} \quad \text { if } p \equiv 1 \quad(\bmod 4), \\
& a_{p^{k}, p^{\ell}}=M(f) \frac{\frac{(-1)^{k \vee \ell}}{p^{2(k \vee \ell)} \frac{1}{1+1 / p^{2}}}}{1-\frac{1}{p^{2}+1}}=M(f) \frac{(-1)^{k \vee \ell}}{p^{2(k \vee \ell)}} \quad \text { if } p \equiv 3 \quad(\bmod 4) .
\end{aligned}
$$

Therefore $a_{q_{1}, q_{2}}=M(f) \chi\left(\left\{q_{1}, q_{2}\right\}\right) /\left\{q_{1}, q_{2}\right\}^{2}$ holds if $\left(q_{1}, q_{2}\right)=\left(p^{k}, p^{\ell}\right)$ where $p \in \mathcal{P}$. Since the function $\left(q_{1}, q_{2}\right) \mapsto a_{q_{1}, q_{2}} / M(f)$ is multiplicative, we obtain the desired result. This completes the proof of Example 3.13.

Acknowledgment. The author sincerely thanks the referee, whose comments and suggestions essentially improved this paper.

\section{References}

[De76] H. Delange, On Ramanujan expansions of certain arithmetical functions, Acta. Arithmetica, 31 (1976) 259-270.

[Ha21] G.H. Hardy, Note on Ramanujan's trigonometrical function $c_{q}(n)$, and certain series of arithmetical functions, Proc. Cambridge Phil. Soc., 20 (1921) 263-271.

[HT16] T. Hilberdink and L. Tóth, On the average value of the least common multiple of $k$ positive integers, arXiv:1604.04508, 2016.

[Rm13] M. Ram Murty, Ramanujan series for arithmetical functions, Hardy-Ramanujan Journal, 36 (2013) $21-33$. 
[Ra18] S. Ramanujan, On certain trigonometrical sums and their applications in the theory of numbers, Transactions of the Cambridge Phil. Society, 22 (1918) 179-199.

[SS94] W. Schwarz and J. Spilker, Arithmetical Functions, Cambridge Univ. Press, 1994.

[Si89] R. Sivaramakrishnan, Classical Theory of Arithmetic Functions, Marcel Dekker, 1989.

[To14] L. Tóth, Multiplicative Arithmetic Functions of Several Variables, a Survey, in vol. Mathematics Without Boundaries, Surveys in Pure Mathematics, Th. M. Rassias, P. Pardalos (Editors), Springer, New York, 2014, 483-514.

[Us07] N. Ushiroya, On a mean value of a multiplicative function of two variables, Probability and Number Theory Kanazawa 2005, Adv. Studies in Pure Math., 49 (eds. S. Akiyama, K. Matsumoto, L. Murata H. Sugita), (2007) $507-515$.

[Us12] N. Ushiroya, Mean-value theorems for multiplicative arithmetic functions of several variables, Integers, 12 (2012) 989-1002.

Noboru Ushiroya

National Institute of Technology, Wakayama College,

77 Noshima Nada Gobo Wakayama, Japan

e-mail: ushiroya@wakayama-nct.ac.jp 